\author{
Proceedings of the $10^{\text {th }}$ International Conference on Applied Informatics \\ Eger, Hungary, January 30-February 1, 2017. pp. 81-94 \\ doi: 10.14794/ICAI.10.2017.81
}

\title{
Efficiency of wavelet transform for detecting manufacturing faults of tapered roller bearings verified by image processing
}

\author{
Krisztián Deák \\ Faculty of Engineering University of Debrecen \\ deak.krisztian@eng.unideb.hu
}

\begin{abstract}
A technique based on decomposition using wavelet transform has been proposed for detecting taperred roller bearing outer race defects and measuring its width. Experiments and subsequent analysis reveal that decomposition of vibration signal with Symlet wavelets is suitable for bearing fault analysis because it improves signal sharpness and suprass noise. Sharp and high amplitude impulses are obtained when roller crosses over the entry and exit of the defect. When the roller remains in contact with the groove base between entry and exit, the impulses due to roughness of the groove surface are observed. The defect evaluation technique using decomposition extracts roller entry and exit components from the signal with good resolution offering a way for feature extraction. The proposed technique has been successfully implemented for measuring defect width. Results has been also verified by image analysis. The maximum deviation between the calculated and the verified value is low enough to prove the right choice of the feature extraction used in the research.
\end{abstract}

Keywords: wavelet, condition monitoring, bearing vibration analysis

\section{Introduction}

Signal analysis is a crucial part of technical diagnostics. Different systems and applications require unique approaches to solve the problem. The purpose of this paper is to present an algorithm based on wavelet transform that is able to reveal grindig marks in manufacturing of taperred roller bearings. To insure the quality of the product is very important but it is not defined in the publications what kind of method is the best solution to the fault identification. It largely depends on both the type of the bearings and the faults. 
Envelope method were applied by Patel et al. [1] for fault diagnosis of deep groove ball bearings and reveal local faults on the races.

Kalman and $\mathrm{H}$ filter was applied by Khanam et al. [2] to measure bearing faults especially in noisy condition with low signal-to-noise ratio value when difficult to identify the useful components of the vibration signal.

A combination of acoustic emission analysis with the traditional vibration analysis was applied by Al-Ghamd and Mba [3]. Traditional vibration analysis with accelaration measurement for the fault detection of slow speed bearings was used by Elforjani et al. [4].

Wavelet analysis was used by Sawalhi and Randall [5] to determine the fault size of the bearings from the vibration signal. They could determine the fault dimension from the entry and exit impulse using two different methods. Feature extraction from the signal is difficult especially when there is a mismatch between direction of defect and the roller-outer race line contact while passing over the defect.

Wavelets are perfect tools for fault feature extraction, singularity detection for signals, denoising and extraction of the weak signals, compression of vibration signals and the system identification. These applications were presented by Peng and Chu [6]. Discrete wavelet transform with Daubechies 4 mother wavelets were applied by Prabhakar et al. [7] to analyse combination of different faults on the races of ball bearings.

A combination of envelope spectrum and wavelet transform and for extraction of defect problems in bearings was used by Shi et al. [8].

Demodulation of vibration signals generated by defects in rolling element bearings by complex shifted morlet wavelets was presented by Nikolaou et al. [9].

Wavelet filter based de-noising is suitable method for detection of weak signatures in bearing fault diagnosis, this type of experiment was made by Qiu et al. [10].

An application of impulse respone wavelet for roller bearing fault detection was presented by Jusheng et al. [11]. Symlet wavelets were used in study of Kumar et al. [12] who analysed taper roller bearings to exactly determine the fault size in the outer ring and presented a detailed study of the decomposition of the vibration signals by discrete wavelet transform with Symlet- 5 .

Symlet wavelest are efficient tool for noise reduction in ECG signals because it could filter out the useful components of the complex signal by Awal et al. [13].

Analytical Wavelet Transform (AWT) based acoustic emission technique for identifying inner race of radial ball bearing was used by Kumar et al. [14].

Multi-scale envelope spectrogram was presented by Yan and Gao [15] to reveal localized structural defects of the bearings and analyzed the vibration signature to exactly determine the existance of the fault on the bearing elements.

An analytical model and simulation to predict the effect of a localized defect on the ball bearing vibrations was presented by Patil et al. [16] considering the contact between the ball and the races as non linear springs.

Envelop analysis was presented in [17] when the vibration signal is first passed through a band-pass filter to obtain a signal without significant noise then Hilbert 
transform is used to get the envelope curve.

Efficient simulations and signal processing techniques to track the spall size was written by Sawalhi and Randall [18].

Discrete wavelet transform of vibration signal to determine the outer race defect width measurement in taper roller bearing was presented by Kumar and Manpreet [19]. Machine learning methods for optimization of parameteres such as support vector machines were used by Mankovits et al. [20].

\section{Feature extraction from the vibration signal}

Wavelet analysis is an advanced tool in signal processing that has attracted much attention. Its use has increased rapidly in communications, image processing and optical engineering applications as an alternative to the Fourier transform in preserving local, non-periodic and multiscaled phenomena. The difference between wavelets and Fourier transforms is that wavelets can provide the exact locality of any changes in the dynamical patterns of the sequence, whereas the Fourier transforms concentrate mainly on their frequency. Moreover, Fourier transform assume infinite-length signals, whereas wavelet transforms can be applied to any kind and size of time series, even when these sequences are not homogeneously sampled in time. In general, wavelet transforms can be used to explore, denoise and smoothen time series, aid in forecasting and other empirical analysis. The basic aim of wavelet analysis is both to determine the frequency (or scale) content of a signal and to assess and determine the temporal variation of this frequency content. Wavelet analysis is an effective tool of condition monitoring in maintenance engineering before machine repairing. It could reveal the basic machine problems in time-frequency domain which is more advantegous than the traditional time methods and frequency methods. Because of its flexibility and computational efficiency several studies presented it in bearing fault diagnosis.

The original signal $s(t)$ passes through a set of low pass and high pass filters emerging as low frequency (approximations, ai) and high frequency (details, di) signals at each decomposition level $n$. Therefore, the original signal $s(t)$ can be written as:

$$
s(t)=a_{n}+\sum_{n=1}^{\infty} d_{i} .
$$

The functions and scales are represented as an approximations value. The curves of scale functions can be modified by the scale parameter, which is the inverse ratio to frequency. The DWT analysis is made by passing the signal through a series of filters. These filters include both high-pass filters and low-pass filters. The high-pass filters examine the high frequency bands called Details ( $\mathrm{Dj}$ ). On the other hand, the low-pass filter analyzes the low frequency band called Approximations (Aj). The Symlets, proposed by Daubechies as modifications to the dbN family, are compactly supported wavelets with least asymmetry and highest number of vanishing moments for a given support width. The symlets are proposed by 
Daubechies as modifications to the db family. The properties of the two wavelet families are similar. The Symlet wavelet is a near symmetrical and linear phase filter, which makes it easier to deal with the small discontinuity present in the signal without any major loss of information which helps in properly locating the point of commencement and exit of roller from the groove defect. The perfect reconstruction and cancellation capability allows them to be used in both continuous wavelet transform and discrete wavelet transform. The use of symlet-5 wavelet based decomposition suitably locates the entry event at the leading edge and impact at the trailing edge, thus giving an indication of the defect size.

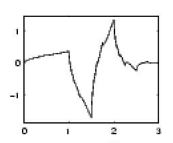

sym2

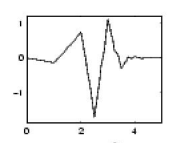

$\operatorname{sym} 3$

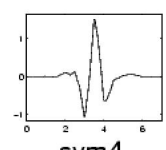

sym4

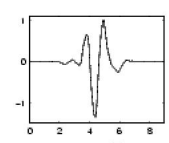

sym5

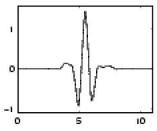

sym6

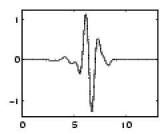

$\operatorname{sym} 7$

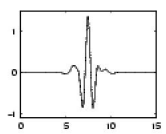

sym8

Figure 1: Symlet wavelets for signal analysis

A multiresolution analysis (MRA) is the design method of most of the practically relevant discrete wavelet transforms (DWT). Wavelet analysis is the breaking up of a signal into shifted and scaled versions of the original (or mother) wavelet. In wavelet analysis, the use of a fully scalable modulated window solves the signalcutting problem. The window is shifted along the signal and for every position the spectrum is calculated. Then this process is repeated many times with a slightly shorter (or longer) window for every new cycle. In the end, the result will be a collection of time-frequency representations of the signal, all with different resolutions. Because of this collection of representations we can speak of a multiresolution analysis.

It uses the series of lowpass and highpass filters (Figure 2). They are usually finite impulse response (FIR) filter is a filter whose impulse response (or response to any finite length input) is of finite duration, because it settles to zero in finite time. This is in contrast to infinite impulse response (IIR) filters, which may have internal feedback and may continue to respond indefinitely (usually decaying).

The defect size on the outer race of the bearing can be estimated from the knowledge of duration between the entry and exit events extracted from the signal after decomposition for the symlet-5 wavelet. Also the peak of impulse would have appeared when the ball might have physically come out from the spall and not when it might have come in contact with the exit corner of the spall. De-stressing and re-stressing phenomena at the entry and exit from the groove defect. The peaks in signal with negative and positive sign describe position of the ball in the groove. The duration between set of events at the leading and trailing of the defect edge is estimated using Symlet based decomposition, fundamental train frequency 


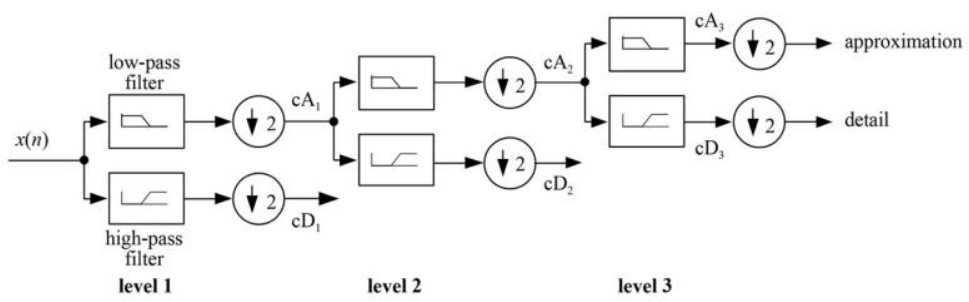

Figure 2: Theoritical way of the multiresolution wavelet analysis with low-and high pass filters

(FTF) and average outer race inner diameter of the bearing (DOI). This method is vital important and advantegous for technical applications because the defect size can be calculated without optical measurements.

The outer race defect width $L_{O D}$ is [19]:

$$
L_{O D}=\pi \cdot \delta t \cdot D_{O I} \cdot F T F
$$

\section{Experiment}

In this study an experimental test rig (Figure 3) has been constructed to measure properly vibration acceleration signatures of the tapered roller bearings. The aim is to create a model to simulate the proper conditions for the measurements focusing on the most efficient signal analysis and feature extraction. Measurement of bearings built in real machines is nearly impossible because of safety reasons. Otherwise, machines in the industry create high vibration level which suprass the useful content of the signal and makes the feature extraction very complicated.

The shaft in the test rig is supported by two taper roller bearings. The one under investigation is No. 30205 tapered roller bearing. Four tapered roller bearings with different defect width on the outer race (OR) were investigated (Table 1) in this experiment. Defect on the outer race is a typical rectangular shape grinding defects (Figure 4) from manufacturing. The shaft is driven by an alternating current motor of $0.75 \mathrm{~kW}$ (made by Cemer), frequency of $50 \mathrm{~Hz}$, and nominal speed of $2770 \mathrm{rpm}$ which is reduced to $1800 \mathrm{rpm}$ with variable speed drive device. Rubber $\mathrm{V}$-belt between the electric engine and the shaft provides smooth running and low vibration which help accurate and precise measurements. Rubber bumpers were installed to reduce vibration of the electric motor to the bearing housing in order to minimalize harmful vibrations. The arrangement provides option of different speeds controlled by Schneider ATV32HU22M2 variable speed drive device.

NI 9234 dynamic signal acquisition is used in the experiments with 4-channels to vibration measurements from integrated electronic piezoelectric (IEPE) and nonIEPE sensors. The NI 9234 delivers $102 \mathrm{~dB}$ of dynamic range. Input channels simultaneously digitize signals at rates up to $51.2 \mathrm{kHz}$ per channel with built-in anti-aliasing filters [21]. PCB IMI 603C01 vibration transducer is used which is an 
industrial type platinum stock piezoelectric sensor with low noise level, sensitivity of $100 \mathrm{mV} / \mathrm{g}$ and frequency range of 0.27 to $10 \mathrm{kHz}$ with top exit 2-pin connector [22]. The accelerometer is placed right above the previously ground surface of the top of the bearing house with screw mechanism perpendicular to the axis of the rotation of the shaft. 32 bit AMD Athlon II X2 M300 2.0 GHz processor is used for data processing which is carried out in Matlab and Labview environment. For visual validation of the defect sizes on the bearing rings Garant MM1-200 video microscope is applied that is an incremental measuring system, built-in image processing with 1.3 megapixel colour camera. Furthermore, Mahr MMQ 200 with precision roundness measuring axis, motorized vertical and horizontal measuring axis is used for roundness deviation measurement to determine both width and depth of the grinding marks.
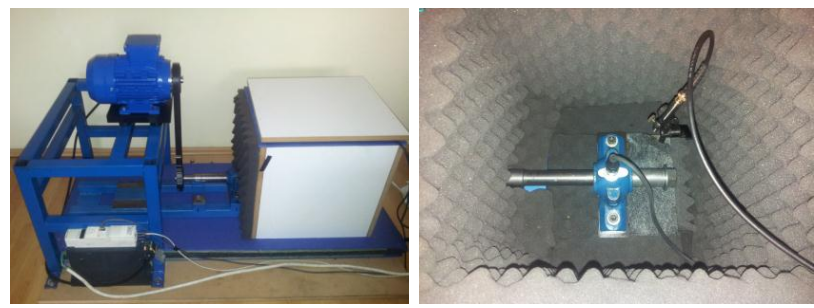

Figure 3: Test rig for tapered roller bearing measurement (left) and its acoustic chamber (right)

Geometrical parameteres of grinding defects of outer rings (OR) made by optical and contact measurements

\begin{tabular}{|c|c|c|c|c|}
\hline Type/Data & OR1 defect & OR2 defect & OR3 defect & OR4 defect \\
Width $[\mathrm{mm}]$ & 0.6311 & 1.2492 & 1.4751 & 1.6236 \\
\hline Depth $[\mu \mathrm{m}] 3$ & 6.5 & 33.6 & 42.3 & 51.4 \\
\hline
\end{tabular}

Table 1

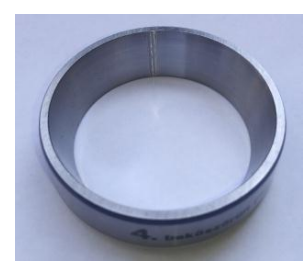

Figure 4: Test rig for tapered roller bearing measurement (left) and its acoustic chamber (right)

Multiresolution wavelet analysis was made down to 4 th level in order to get precise frequency analysis. 


\section{Result and discussion}

Bearing defects generate transients in the vibration signal when the rollers pass through the defects. The fault frequencies can be calculated by numerical way. FTF (fundamental train frequency) is $12.88 \mathrm{~Hz}$ in case of $18001 / \mathrm{min}$ that was applied in the experiment.

$$
L_{O D}=\pi \cdot \delta t \cdot D_{O I} \cdot F T F=1713.4 \cdot \delta t .
$$

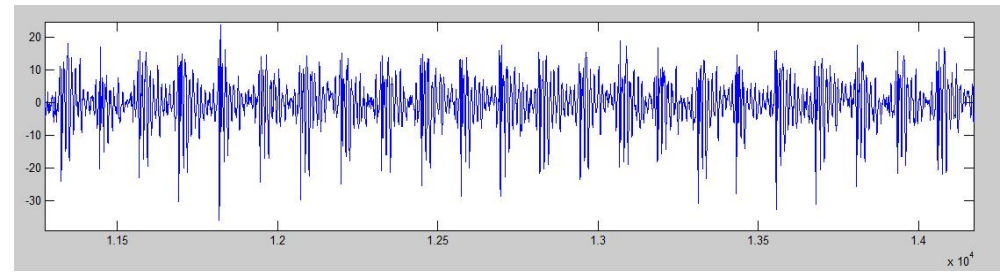

Figure 5: Typical raw time domain signal of bearing having 0.6311 $\mathrm{mm}$ of ground fault width on the outer race

In order to get the frequency spectra of the faulty bearings Fourier transform was applied for all bearings in the experiment. Figure 5 shows the time-domain of the measured signal of outer race defect of $0.6311 \mathrm{~mm}$ with $5 \mathrm{~ms}$ repetive transient impulses and Figure 6 represents its spectra. The highest energy content of the transient impulse occurs at $2.09 \mathrm{kHz}$.

On the basis of the Fourier analysis it can be determined that down to 4th level wavelet multiresolution analysis of the raw vibration signal is recommended as Figure 7 shows. Higher decomposition is not necessary because it might not reveal any further information of the signal. Regarding the BPFO frequency analysis was made at 3th detail level (cD3) from 1.25 to $2.5 \mathrm{kHz}$ where the transient impulse of $2.09 \mathrm{kHz}$ is found.

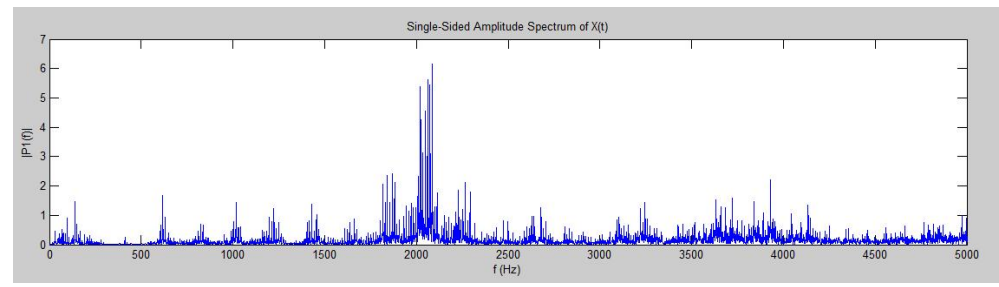

Figure 6: Frequency domain spectrum of bearing having 0.6311 $\mathrm{mm}$ of ground fault width on the outer race

Figures 7-9 show the decomposition graph of the multiresolution analysis (MRA) of the $0.6311 \mathrm{~mm}$ fault width down to 4th level. Signals were acquired and processed for other defects in the similar manner. In the experiment 20 impulses were 
measured and the average time values of the entry, de-stressing and exit point of all of the four outer rings were determined for precise calculation that means 240 time data.

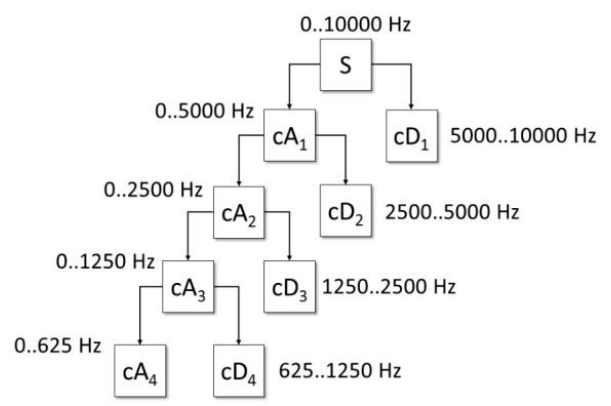

Figure 7: Multiresolution analysis (MRA) of the original vibration signal down to 4 th level

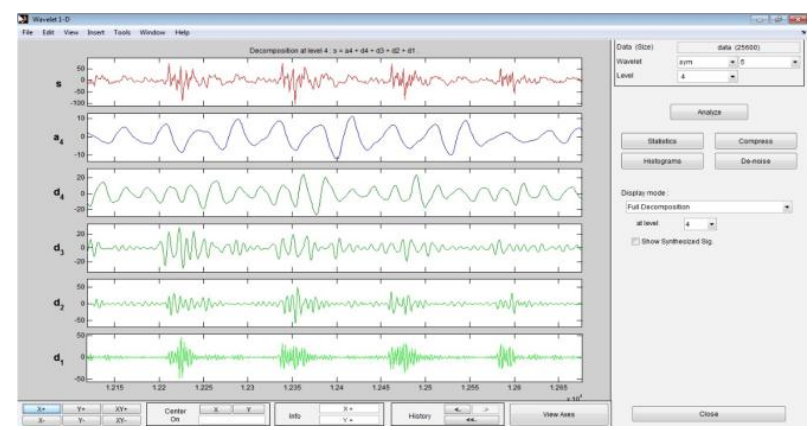

Figure 8: Full decomposition graph of the original vibration signal of $0.6311 \mathrm{~mm}$ fault width down to 4 th level

To determine the entry point higher value of de-stressing is preferred. The decomposition graph down to 4th level has been drawn by using Symlet-5 mother wavelet for the case having defect width $0.6311 \mathrm{~mm}$ in the outer race which is shown in Figure 7. Three points in the signal namely A, B and C are shown in the Figures 10-11 represent the corners of the defect and maximum de-stressing point respectively. Three different positions of the roller with respect to points $\mathrm{A}, \mathrm{B}$ and $\mathrm{C}$ at a cross section are highlighted with circles in Figure 10. Point $\mathrm{A}$ is the entry point of the roller into the outer race defect, $\mathrm{B}$ is the maximum de-stressing, $\mathrm{C}$ is the exit point. Spotting A from raw signal is difficult because there is no significant variation in amplitude but it can be increased by using wavelet decomposition graph. At point B, the roller strikes the defect base with high impact which results in re-stressing phenomena and high energy content in the signal. After this event the roller remains in contact with the defect base for 


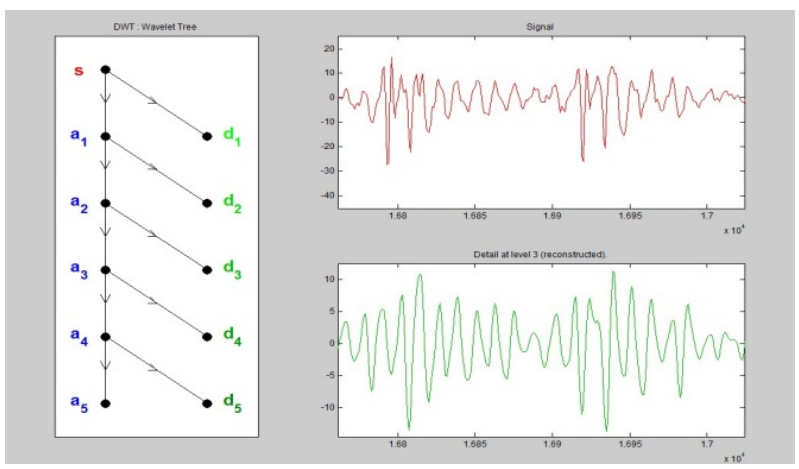

Figure 9: Tree decomposition graph of the original vibration signal of $0.6311 \mathrm{~mm}$ fault width down to 4th level, two transient impulses are highlighted

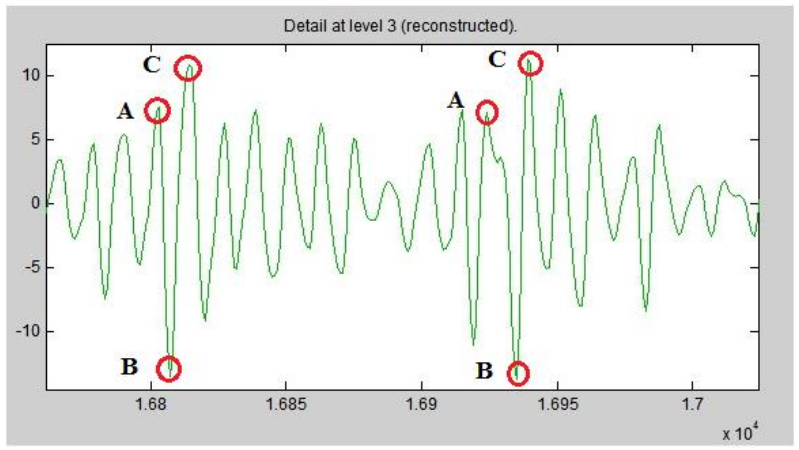

Figure 10: Detail graph at 3rd level of the MRA of the original vibration signal of $0.6311 \mathrm{~mm}$ fault width with two typical transient impulses

a while and during this period impulses are observed due to the rough surface of defect. When the roller comes in contact with the point $\mathrm{C}$ namely the edge of the defect it again generates high amplitude in the signal and beyond this point it progressively decreases in amplitude. It is called as re-stressing event.

The de-stressing value from point $\mathrm{A}$ to point $\mathrm{B}$ has been calculated in percentage of the range for each individual transient impulses in the 4 th level decomposition graph obtained through Symlet-5 and raw signal graph. De-stressing values are calculated in percentage of the range of the transient impulses. In this experiment Symlet-6 gives only 36\% de-stressing to the full range of amplitude of the burst while the Symlet-5 gives $41 \%$ de-stressing in case of defect width $0.6311 \mathrm{~mm}$. Decomposition by Symlet-4 makes the signal skewed and pointed at the tip which is a deviation from the actual one that means it is not appropriate for fault monitoring. In case of Symlet-5, the average value of de-stressing in raw signal is only $7.48 \%$ 


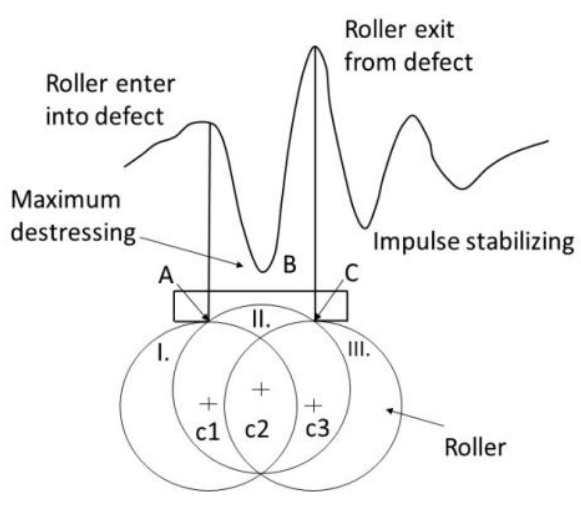

Figure 11: Analyis of the transients impulse presenting the entry and exit points of the roller into the grinding defects on the outer ring

and in the decomposition graph is $50.43 \%$. Symlet-5 wavelet is appropriate for fault detection it could spot the point A by modulating the signal. Between points $\mathrm{A}$ and $\mathrm{C}$ there is no direct contact with the defect base because no intermediate peak detected in the signal. Finally, the signal starts stabilizing as its amplitude progressively decreases due to damping behaviour of elastic material of the bearing creating harmonic oscillation. To obtain the time taken by roller to pass the groove defect data points are calculated between the points $\mathrm{A}$ and $\mathrm{C}$.

As the bearing in the experiment has 16 rollers the same number of successive bursts are calculated for estimating the time taken by roller to pass over the outer race defect. Further using Eq. (3) which has been simplified for the specified bearing at the speed $1800 \mathrm{rpm}$ (i.e. $\mathrm{FTF}=12.88 \mathrm{~Hz}$ ) the defect width is calculated. The defect width is also evaluated and therefore validated by image analysis. The values of defect have been measured by making use of signal processing as well as image processing. It has been observed that the variation in data points between bursts is more at low defect widths when shaft is not loaded. The maximum difference in result has been obtained to be $2.41 \%$ for defect width of $0.6311 \mathrm{~mm}$.
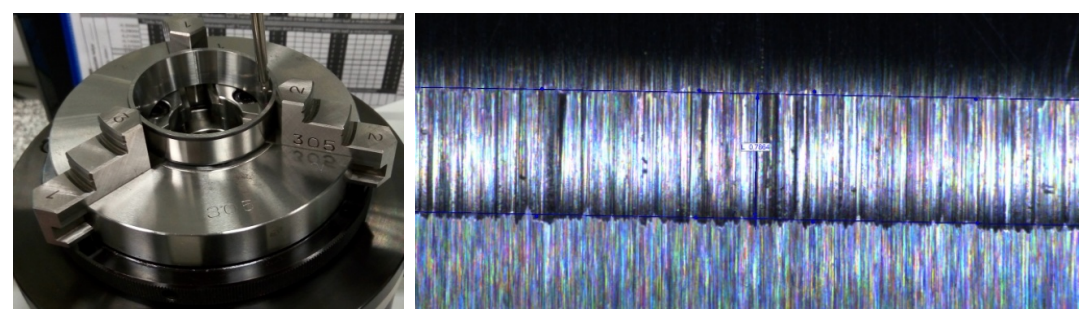

Figure 12: Optical measurement of the outer race defect width with Garant MM1-200 video microscope (left) and its image (right) 
To find the edges of the grinding marks on the outer race made by the Garant MM1-200 video microscope edge detection was used which is basically the name for a set of mathematical methods which aim at identifying points in a digital image at which the image brightness changes sharply or, more formally, has discontinuities. The Gaussian filter was used for noise reduction. Intensity gradient of the image was found a pair of convolution masks were used in $\mathrm{x}$ and $\mathrm{y}$ directions. Gradient strength and direction were found with the following way. Non-maximum suppression is applied. This removes pixels that are not considered to be part of an edge. Hence, only thin lines (candidate edges) will remain. The final step was hysteresis. Canny edge detection algorithm uses two thresholds (upper and lower). If a pixel gradient is higher than the upper threshold, the pixel is accepted as an edge. If a pixel gradient value is below the lower threshold, then it is rejected. If the pixel gradient is between the two thresholds, then it will be accepted only if it is connected to a pixel that is above the upper threshold.
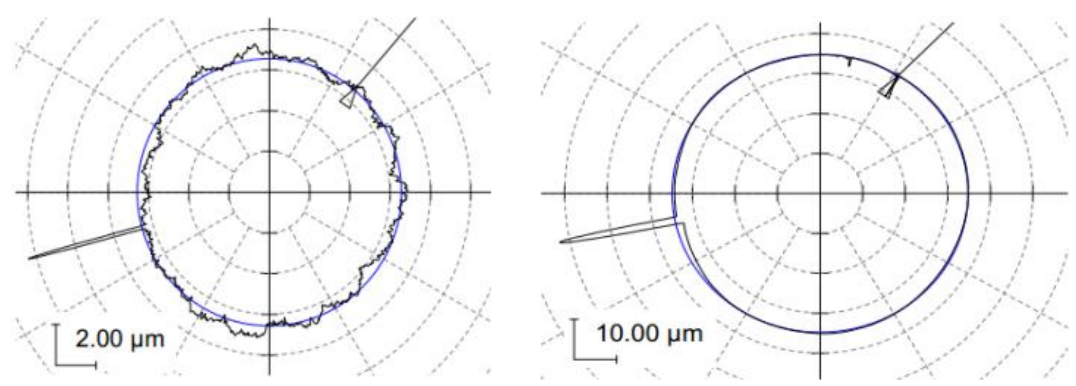

Figure 13: Result of the roundness measurement of the outer race defect with Mahr MMQ 200 equipment, OR1 defect (left) and OR2 defect (right)
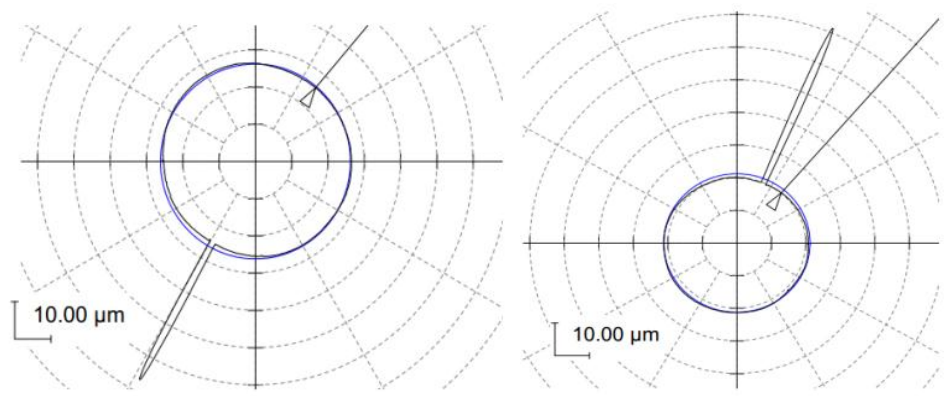

Figure 14: Result of the roundness measurement of the outer race defect with Mahr MMQ 200 equipment, OR3 defect (left) and OR4 defect (right) 


\begin{tabular}{|c|c|c|c|}
\hline Case / Parameter & $\begin{array}{c}\text { Calculated } \\
\text { defects } \\
\text { width from } \\
\text { points }(\mathrm{mm})\end{array}$ & $\begin{array}{c}\text { Optically } \\
\text { measured } \\
\text { defect } \\
\text { width }(\mathrm{mm})\end{array}$ & $\begin{array}{c}\text { Deviation } \\
\text { between } \\
\text { calculated and } \\
\text { measured } \\
\text { data }(\%)\end{array}$ \\
\hline OR1 defect & 0.6462 & 0.6311 & 2.41 \\
\hline OR2 defect & 1.4481 & 1.4751 & 1.83 \\
\hline OR3 defect & 1.2685 & 1.2492 & 1.56 \\
\hline OR4 defect & 1.6391 & 1.6236 & 0.92 \\
\hline
\end{tabular}

Table 2: Geometrical parameteres of grinding defects by optical and contact measurements

\section{Conclusion}

Tapered roller bearings are important parts of all rotary machines. Defects of bearings can be derived from either manufacturing or operation. Grinding marks on the outer rings are analyzed to determine their width. Wavelet method is more efficient for detecting sharp edges and fast changes in signal than conventional frequency domain method. Symlet-5 wavelet has linear phase nature therefore sudden changes in the vibration signal could be detected efficiently. Experiments were carried out on a test-rig which is capable of measuring tapered roller bearings under different conditions by creating an efficient model for feature extraction. The proposed method has been succesfully implemented to reveal manufacturing problems. Defect width has been detected with maximal deviation of $2.41 \%$ from the defect width which was verified using visual techniques and image analysis with edge detection.

\section{References}

[1] Patel, V. N., Tandon, N., And Pandey, R., K., Defect Detection in Deep Groove Ball Bearing, Presence of External Vibration Using Envelope Analysis and Duffing Oscillator, Measurement, 45, (2012), 960.

[2] Khanam, S., Tandon, N., Dutt, J. K., Fault Identification of Rolling Element Bearings from Vibration Signals, An Application of Kalman and H Filters. LOWHUV 10th International Conference on Vibrations in Rotating Machinery (VIRM10), IMechE London, UK, Woodhead Publishing Limited, Institution of Mechanical Engineers (IMechE), (2012), 703-713.

[3] Al-Ghamd, A.M., Mba, D., A Comparative Experimental Study on the Use of Acoustic Emission and Vibration Analysis for Bearing Defect Identification and Estimation of Defect Size, Mechanical Systems and Signal Processing, (2006), 1537.

[4] Elforjani, M., Mba, D., Accelerated Natural Fault Diagnosis in Slow Speed Bearings with Acoustic Emission, Engineering Fracture Mechanics 77, (2010), 112. 
[5] Sawalhi, N., Randall, R.B., Vibration Response of Spalled Rolling Element Bearings: Observations, Simulations and Signal Processing Techniques to Track the Spall Size, Mechanical Systems and Signal Processing 25, (2011), 846.

[6] Peng, Z.K., Chu, F.L., Application of the Wavelet Transform in Machine Condition Monitoring and Fault Diagnostics, A Review with Bibliography, Mechanical Systems and Signal Processing 18, (2004), 199.

[7] Prabhakar, S., Mohanty, A.R., Sekhar, A.S., Application of Discrete Wavelet Transform for Detection of Ball Bearin g Race Faults, Tribology International 35, (2002), 793.

[8] Shi, D. F., Wang, W. J., And Qu, L. S., Defect detection for bearings using envelope spectra of wavelet transform, ASME Journal of Vibration and Acoustics, 126 (4), (2004), 567.

[9] Nikolaou, N. G., Antoniadis, I. A., Demodulation of Vibration Signals Generated by Defects in Rolling Element Bearings Using Complex Shifted Morlet Wavelets, Mechanical Systems and Signal Processing 16(4), 2002, 677.

[10] Qiu, H., Lee, J., Lin, J., Yu, G., Wavelet Filter-Based Weak Signature Detection Method and its Application on Rolling Element Bearing Prognostics, Journal of Sound and Vibration 289, (2006), 1066.

[11] Junsheng, C. , Dejie, Y., Yu, Y., Application of an Impulse Response Wavelet to Fault Diagnosis of Rolling Bearings, Mechanical Systems and Signal Processing 21, (2007), 920.

[12] Kumar, R., Singh, M., Outer Race Defect Width Measurement in Taper Roller Bearing using Discrete Wavelet Transform of Vibration Signal, Measurement 46, (2013), 537.

[13] Awal, M. A., Mostafa, S. S., Ahmad, M., 2012. Quality Assessement of ECG Signal Using Symlet Wavelet Transform, Proceedings of International Conference on Advances in Electrical Engineering, pp.129-134.

[14] R. Kumar, D.P. Jena, M. Bains, Identification of inner race defect in radial ball bearing using acoustic emission and wavelet analysis, in: Proceedings of ISMA 2010 including USD 2010 Leuven (Belgium), 2010, pp. 2883-2891.

[15] R. YAn, R.X. GAO, Multi-scale enveloping spectrogram for vibration analysis in bearing defect diagnosis, Tribology International 42 (2009) pp. 293-302.

[16] M.S. Patil, J. Mathew, P.K. Rajendrakumar, S. Desai, A theoretical model to predict the effect of localized defect on vibrations associated with ball bearing, International Journal of Mechanical Sciences 52 (9) (2010) pp.1193- 1201.

[17] W. He, Z. Jiang, K. Feng, Bearing fault detection based on optimal wavelet filter and sparse code shrinkage, Measurement 42 (2009) pp.1092-1102.

[18] N. SaWAlhi, R.B. Randall, Vibration response of spalled rolling element bearings: observations, simulations and signal processing techniques to track the spall size, Mechanical Systems and Signal Processing 25 (2011) pp.846-870.

[19] Rajesh Kumar, Manpreet Singh, Outer race defect width measurement in taper roller bearing using discrete wavelet transform of vibration signal, Measurement 46 (2013) pp.537-545 
[20] Mankovits, T., Szabó, T., Kocsis, I., PÁczelt, I., Optimization of the Shape of Axi-Symmetric Rubber Bumpers, Strojniski vestnik-Journal of Mechanical Engineering, 60, no. 1, (2014), 61-71.

[21] NI 9234 DATA ACQUisition MOdule, http://www.ni.com/datasheet/pdf/en/ $\mathrm{ds}-316$

[22] PCB IMI 603C01 TRANSDUCER, https://www.pcb.com/contentstore/docs/ PCBCorporate/IMI/Products/Manuals 